\title{
New approach for cardiac insulin resistance assessment using nuclear imaging: Moving research closer to practice
}

\author{
Kuan-Yin Ko, a,b and Yen-Wen $\mathrm{Wu}^{\mathrm{c}, \mathrm{d}, \mathrm{e}}$ \\ a Department of Nuclear Medicine, National Taiwan University Cancer Center, Taipei, Taiwan \\ b Graduate Institute of Clinical Medicine, College of Medicine, National Taiwan University, \\ Taipei, Taiwan \\ c Department of Nuclear Medicine, Far Eastern Memorial Hospital, New Taipei City, Taiwan \\ d Division of Cardiology, Cardiovascular Medical Center, Far Eastern Memorial Hospital, New \\ Taipei City, Taiwan \\ e National Yang-Ming University School of Medicine, Taipei, Taiwan
}

Received Jan 29, 2021; accepted Jan 29, 2021

doi: 10.1007/s12350-021-02566-1

See related article, pp. 1419-1429

Insulin resistance is considered to be the hallmark of the pathophysiology of type 2 diabetes and metabolic syndrome, and it has been shown to predict the development of cardiovascular diseases. ${ }^{1}$ In order to maintain cardiac contractility and the circulation of blood and oxygen to peripheral organs, the heart needs more energy than any other organ. Fatty acids are the main source of energy in an intact heart, and under stress or pathological conditions the myocardium also utilizes glucose to compensate for energy deficiencies. Insulin signaling may directly control cardiac metabolism; however, its main role may be the regulation of substrate delivery from the periphery to the heart. ${ }^{2}$ In generalized insulin resistance, insulin-mediated glucose transport in cardiac muscle is impaired and associated with the development of heart failure unrelated to myocardial ischemia and hypertension. ${ }^{3,4}$ Many basic studies have underscored the biphasic changes of insulin signaling in the myocardium to systemic metabolic alterations in the evolution of heart failure. In early adaptation of the heart

Reprint requests: Yen-Wen Wu, Department of Nuclear Medicine, Far Eastern Memorial Hospital, New Taipei City, Taiwan; wuyw0502@gmail.com

J Nucl Cardiol 2022;29:1430-3.

$1071-3581 / \$ 34.00$

Copyright (c) 2021 American Society of Nuclear Cardiology. to systemic resistance, the expression of glucose transporter type 4 (GLUT4) can be repressed; however proximal insulin signaling to phosphatidylinositol-3-kinase and protein kinase $B$ may remain intact accompanied by left ventricle (LV) remodeling. However, insulin signaling pathways can become desensitized over time, leading to cardiac decompensation via various mechanisms. ${ }^{5}$ Metabolic imaging with ${ }^{18} \mathrm{~F}$-fluorodeoxyglucose (FDG) PET/CT has been widely used to both assess myocardial viability and also evaluate exogenous glucose utilization rate. Several clinical and animal studies have observed myocardial glucometabolic derangements in patients with diabetes mellitus and heart failure using dynamic imaging, although the causal relationship could not be clearly verified. In addition, myocardial glucose metabolism can be altered by blood substrate levels and some medications for hyperlipidemia and diabetes. ${ }^{6-9}$

Even though insulin resistance is an important determinant in cardiometabolic diseases, the gold standard technique of directly measuring insulin resistance remains the hyperinsulinemic-euglycemic clamp, which is not clinically feasible due to its time-consuming and labor-intensive procedure. ${ }^{10}$ Therefore, a number of surrogate indices such as homeostasis model assessment (HOMA) and quantitative insulin sensitivity check index (QUICKI) have been proposed to simplify and improve the determination of whole-body insulin resistance. ${ }^{11,12}$ In this issue of the Journal of Nuclear Cardiology, Perret et al reported a new radionuclide imaging method with ${ }^{123}$ I-6-deoxy-6-iodo-D-glucose (6DIG) using dynamic 


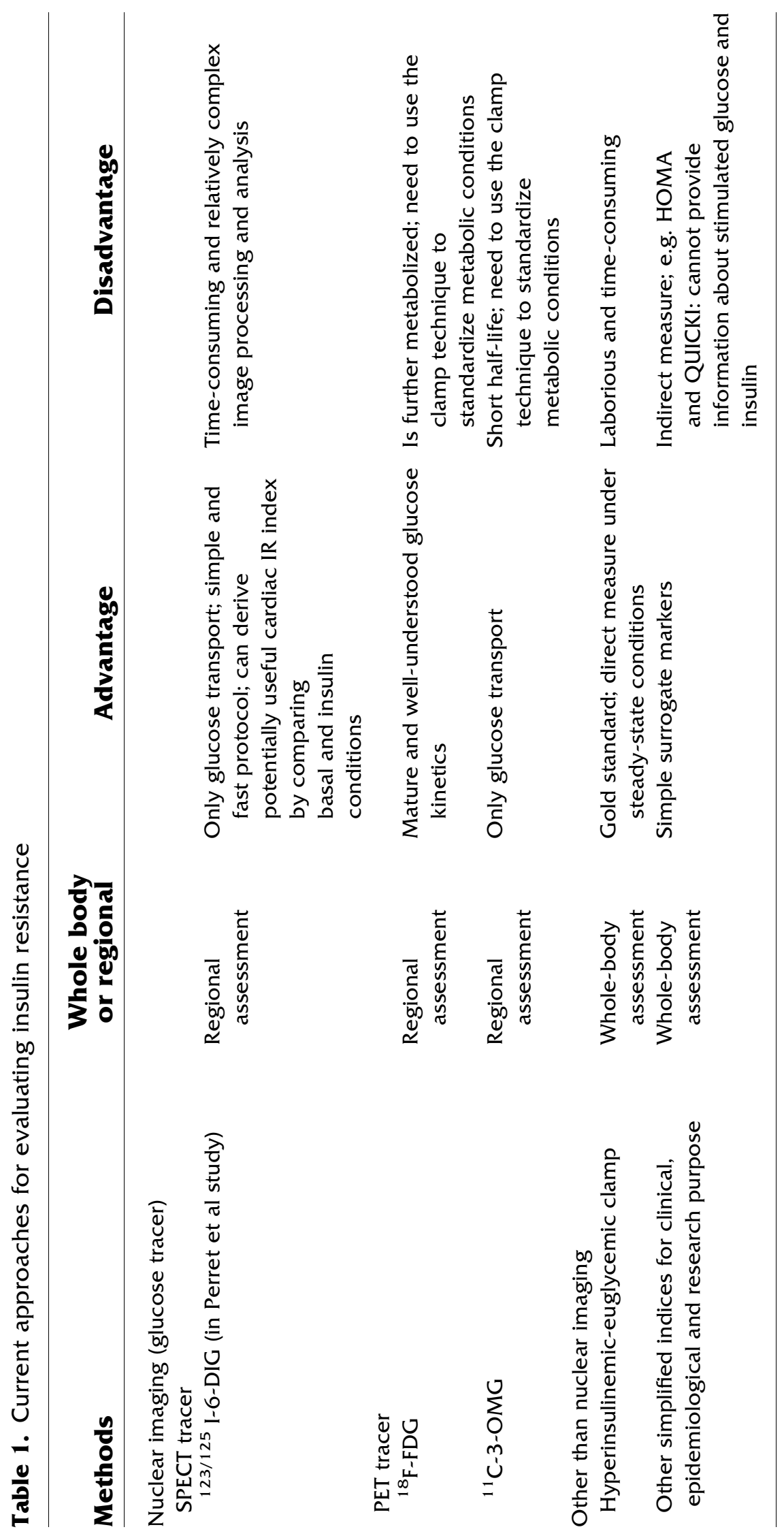


SPECT to assess cardiac insulin resistance. ${ }^{13}$ 6DIG enters the cell using glucose transporters without being metabolized, and therefore it can transport across the cell membrane bi-directionally. Its biological behavior is similar to that of 3-O-methyl-D-glucose (3-OMG) labelled with a short half-life radioisotope $\left({ }^{11} \mathrm{C}, t_{1 /}\right.$ ${ }_{2}=20 \mathrm{~min}$ ), which has been applied but is not clinically routinely used in humans to study regional glucose transport with PET imaging. The potential of 6DIG to assess insulin resistance without the need for a glucose clamp using modelling of 6DIG kinetics acquired by NaI probes has been demonstrated in previous work. ${ }^{14,15}$ However, the protocol remains complex because it requires extracorporeal blood circulation. In the present study, the authors propose a new method with a rapid and simple protocol using cardiac planar images on a $\gamma$ camera under basal and insulin conditions. This translational research was divided into two parts: validation in rats, and the first study in humans. In two wellestablished insulin resistance rodent models, the results clearly demonstrated the stimulation of cardiac 6DIG by insulin in the control groups; however, the effect was not observed in insulin-resistant animals. Moreover, the authors performed experiments to show the reproducibility and sensibility of the derived cardiac insulinresistant index $\left(k(2,1)_{\text {insulin }} / k(2,1)_{\text {basal }}\right.$; coefficient $k(2,1)$ : 6DIG transport from blood to the heart and a comparison of basal versus insulin conditions). In the human study, they studied two groups of healthy volunteers and diabetic patients treated with metformin only. To obtain blood and myocardial kinetics before mathematical modelling, the authors used factor analysis to process imaging sequences based on signal intensity evolution analysis of each pixel over time. The results revealed that cardiac insulin resistance indices were lower in the diabetic patients than in the healthy people, although statistical significance was not reached.

Although there are several approaches to evaluate insulin resistance as shown in Table 1, the significance of the simple and fast tool for research and clinical settings to measure cardiac insulin resistance in Perret et al's study is in providing insights into the role of myocardial insulin resistance in non-ischemic cardiomyopathy. However, it is worth mentioning that similar to FDG, the iodinated glucose tracer could not reflect changes in the activation of signaling intermediates that are downstream of insulin receptors. For example, even when GLUT4 protein levels are normal, impaired intracellular signaling can lead to reduced insulin-mediated cardiac glucose uptake, and intact versus impaired intracellular signaling can impact LV remodelling in distinct ways. In addition, it is not a convenient method in a clinical setting, as manual postimaging processing is still required to obtain cardiac insulin resistance index rather than being a fully automatic operation and analysis process, which increases the cost and time. Regardless of these limitations, the new methodology proposed by Perret et al is encouraging, and may be a powerful tool for precision and individualized medicine. Further validation in larger prospective trials is warranted.

\section{References}

1. Yip J, Facchini FS, Reaven GM. Resistance to insulin-mediated glucose disposal as a predictor of cardiovascular disease. J Clin Endocrinol Metab 1998;83:2773-6.

2. Abel ED, O'Shea KM, Ramasamy R. Insulin resistance: Metabolic mechanisms and consequences in the heart. Arterioscler Thromb Vasc Biol 2012;32:2068-76.

3. Kannel WB, Hjortland M, Castelli WP. Role of diabetes in congestive heart failure: The Framingham study. Am J Cardiol 1974;34:29-34

4. Kannel WB, McGee DL. Diabetes and cardiovascular disease. The Framingham study. JAMA 1979;241:2035-8.

5. Riehle $\mathrm{C}$, Abel ED. Insulin signaling and heart failure. Circ Res 2016;118:1151-69.

6. Nielsen R, Jorsal A, Iversen P, Tolbod L, Bouchelouche K, Sorensen $\mathrm{J}$, et al. Heart failure patients with prediabetes and newly diagnosed diabetes display abnormalities in myocardial metabolism. J Nucl Cardiol 2018;25:169-76.

7. Ko KY, Wu YW, Liu CW, Cheng MF, Yen RF, Yang WS. Longitudinal evaluation of myocardial glucose metabolism and contractile function in obese type 2 diabetic $\mathrm{db} / \mathrm{db}$ mice using small-animal dynamic (18)F-FDG PET and echocardiography. Oncotarget 2017;8:87795-808.

8. Ko KY, Wang SY, Yen RF, Shiau YC, Hsu JC, Tsai HY, et al. Clinical significance of quantitative assessment of glucose utilization in patients with ischemic cardiomyopathy. J Nucl Cardiol 2020;27:269-79.

9. Tsai SY, Wu YW, Wang SY, Shiau YC, Chiu KM, Tsai HY, et al. Clinical significance of quantitative assessment of right ventricular glucose metabolism in patients with heart failure with reduced ejection fraction. Eur J Nucl Med Mol Imaging 2019;46:2601-9.

10. DeFronzo RA, Tobin JD, Andres R. Glucose clamp technique: a method for quantifying insulin secretion and resistance. Am J Physiol 1979;237:E214-23.

11. Matthews DR, Hosker JP, Rudenski AS, Naylor BA, Treacher DF, Turner RC. Homeostasis model assessment: Insulin resistance and beta-cell function from fasting plasma glucose and insulin concentrations in man. Diabetologia 1985;28:412-9.

12. Katz A, Nambi SS, Mather K, Baron AD, Follmann DA, Sullivan G, et al. Quantitative insulin sensitivity check index: A simple, accurate method for assessing insulin sensitivity in humans. J Clin Endocrinol Metab 2000;85:2402-10.

13. Muniyappa R, Lee S, Chen H, Quon MJ. Current approaches for assessing insulin sensitivity and resistance in vivo: Advantages, limitations, and appropriate usage. Am J Physiol Endocrinol Metab 2008;294:E15-26.

14. Briat A, Slimani L, Perret P, Villemain D, Halimi S, Demongeot J, et al. In vivo assessment of cardiac insulin resistance by nuclear probes using an iodinated tracer of glucose transport. Eur J Nucl Med Mol Imaging 2007;34:1756-64.

15. Perret P, Slimani L, Briat A, Villemain D, Halimi S, Demongeot J, et al. Assessment of insulin resistance in fructose-fed rats with 
125I-6-deoxy-6-iodo-D-glucose, a new tracer of glucose transport. Eur J Nucl Med Mol Imaging 2007;34:734-44.
Publisher's Note Springer Nature remains neutral with regard to jurisdictional claims in published maps and institutional affiliations. 